\title{
Anthropometric Measurement Changes in Orthodontic and Orthognathic Surgery Patients in Dravidian Population: A Photographic Retrospective Study
}

\author{
Hemanth Karalapti ${ }^{1}$, Ratna Parameswaran ${ }^{2}$, Nagachandran Kandasamy ${ }^{3}$, Devaki Vijaylakshmi ${ }^{4}$ \\ ${ }^{I}$ Senior Lecturer, Department of Orthodontics and Orthopeadics, Madha Dental College, Chennai, Tamilnadu, \\ India, ${ }^{2}$ Professor, Department of Orthodontics and Orthopeadics, Meenakshi Ammal Dental College, Chennai, \\ Tamilnadu, India, ${ }^{3}$ Professor, Department of Orthodontics and Orthopeadics, Madha Dental College, Chennai, \\ Tamilnadu, India, ${ }^{4}$ Professor and Head of the Department, Department of Orthodontics and Orthopeadics, \\ Meenakshi Ammal Dental College, Chennai, Tamilnadu, India
}

\begin{abstract}
Introduction: To evaluate the anthropometric and divine proportion measurements using pre and posttreatment photographs in patients subjected to only orthodontic treatment and orthodontics with orthognathic surgery.

Materials and Method: The sample size was calculated with a power value of $95 \%$ which yielded a sample size of 126 patients. The pre and post photographs of these patients were divided into two groups namely Group I where patients treated only with orthodontics (64 patients) and Group II where patients were treated by both orthodontics and orthognathic surgery( 52 patients). The Standard Anthropometric facial proportions and divine proportion measurements were used to digitally assess the pre and post-treatment facial changes using Ilexis FACAD software (Version 3.8.00). These photographs were standardized with the help of interpupillary line measurement which was done on both digital photographs and clinically and correlated.

Results: Group I showed statistical significance $(\mathrm{P}<0.005)$ with upper lip length, lower lip length, upper lip, and lower lip vermillion changes whereas Group II subjects showed statistical significance in Skeletal vertical changes. The lower facial height, lower lip length was significant in Class II orthognathic patients whereas the upper facial height, alar basal width, and mouth width showed significant differences $(\mathrm{P}<0.005)$ in Class III orthognathic patients.

Conclusion: Significant changes were noticed in both groups when pre and post-treatment values were compared, but orthognathic subjects comparatively showed higher significance values in vertical parameters which were near to the ideal anthropometric values and divine proportion ratios.
\end{abstract}

Keywords: Anthropometrics, Photographicanalysis, ivineproportions and Orthognathicsurgery.

\section{Introduction}

Facial beauty and physical Esthetics has a strong emphasis on modern social society and individual

\section{Corresponding Author:}

\section{Hemanth Karalapti}

Senior Lecturer, Department of Orthodontics and

Orthopeadics, Madha Dental College, Chennai,

Tamilnadu, India

e-mail: hemanthapparao@yahoo.com well-being. The 18th-century philosopher Alexander Baumgarten, who established esthetics as a distinct field of philosophy, coined the term, which is derived from the Greek word for sensory perception (aisthesis) ${ }^{[1]}$. Aesthetics play a major role in day to day life of the people for overall social and personal happiness. Even though tastes, fashion, and standards of beauty change from time to time, there appears to be certain facial proportions and relationships that provide a basis for the diagnosis and planning for improvement of facial form. 
Many authors over the years tried quantifying facial beauty and physical attractiveness, among which Ricketts ${ }^{[2]}$ gave "golden proportions" through a mathematical proportion of Fibonacci series and Farkas $^{[3]}$ introduced "Anthropometric measurements" based on the extensive technical study of face and head measurements in accordance to age, sex, and ethnic origin. And these indices are referred to quantify and qualify the term overall aesthetics of the face.

In today's society esthetics play an overwhelming role in a variety of media. The facial standards of esthetics bring the perception of beauty and social acceptance ${ }^{[4]}$. In recent times, patients seek treatment with the most common motive being the enhancement of facial beauty through orthodontic and orthognathic procedures.

The present study aimed to compute the facial esthetics using two-dimensional photographs through anthropometric measurements and divine proportions in patients who underwent orthodontic treatment only compared with patients who underwent Orthodontic and Orthognathic surgical treatment. This comparison adds insight to the present literature based on the dramatic changes that can be achieved through Orthodontic and Orthognathic treatment modalities respectively.

The study also aimed to perceive whether the post orthodontic or post orthognathic facial measurements obtained, were near to the ideal anthropometric measurements and divine proportions. The null hypothesis states that there are no changes in the anthropometric measurements when patients are subjected to orthodontic or orthognathic surgery protocols

\section{Materials and Method}

This prospective study was conducted by evaluating records of patients who underwent Orthodontic treatment only and Orthodontic combined with Orthognathic treatment in the Department of Orthodontics and Dentofacial Orthopaedics at our center.

The inclusion criteria for the Orthodontic treatment group (Group I) were patients with dentoalveolar Class I bimaxillary protrusion, dentoalveolar Class II division 1 with an overjet of less than $7 \mathrm{~mm}$, dentoalveolar Class II division 2, and dentoalveolar Class III with an edge to edge incisal relationship. The Orthognathic surgical Group (Group II) comprised of patients who presented with Skeletal Class II division 1 (Sub-group A) with overjet greater than $7 \mathrm{~mm}\left(\mathrm{ANB} \geq 6^{\circ}\right)$ and Skeletal Class III (Sub-group B) with $\mathrm{ANB} \leq-1^{\circ}$.

Patients with gross facial asymmetry, craniofacial deformity, systemic illness, improper records, cleft lip and cleft palate, and patients who required re-treatment and those under treatment progress were excluded from the study.

Based on the power factor of about $95 \%$ the sample size calculated was 128 patients.

The Pre and Post-treatment Frontal view photographs of 116 Patients were collected from the archives in our center over a period of 15 years, of which 64 were in group I (Orthodontics only) and 52 were in group II (Orthodontics and Orthognathic surgery) as only 52 patients records were retrievable. The sample size was kept to 116 patients as we narrowed it to only the Dravidian population who underwent orthodontic treatment and orthodontic combined with orthognathic surgery exclusively in our center. The subjects who underwent orthodontic therapy were randomly selected relative to the other group. The patient's age ranged from 13 to 35 years(Mean -25.1yrs). Pre-treatment and Post-treatment photographs were obtained using a Digital camera (Nikon D3200, Japan) fitted with a Ring Flash (Nissin MF18) onto a tripod stand with the object to operator's distance measuring to 5 feet 8 and a standardized zoom of $105 \mathrm{~mm}$. The sample allocation was done on a randomized method in individual groups.

A Digital Photographic album was created for both the groups and each album consisted of Pre and Posttreatment photographs which were paired and were placed in a random sequence and assessed for change in proportions.

The images were imported into the computerized software (FACAD AB-2014 software, Version 3.8.0.0, Sweden) by a single operator. The standardization and calibration of the photographs were done by correlating the digitized inter-pupillary measurement from the photographs with the clinical measurement of interpupillary width in patients. The inter-pupillary distance was used as a stable facial reference as it is not subjected to any change after the age of 11years for girls and 15 years for boys. ${ }^{[7]}$ The Inter-pupillary width was calculated, from the mid-point of the pupil of each eye or mid-point of each orbit on either side ${ }^{[9]}$.

Twenty-seven parameters of the Anthropometric 
proportion5 were used for the analysis (Table I, Table II, Table III) since the software which was employed for this study could digitize parameters that were in the 2D frontal view. Using the analysis, 116 patient's pre and post-treatment facial proportional changes were measured and evaluated.

Statistical Analysis: The results are statistically analysed and tabulated using SPSS software (produced by SPSS Inc software house in Delaware and incorporated into IBM in 2010, United States of America). The unpaired student ' $t$ ' test was used to compare the data between the groups and the paired student t-test was used within the groups.

\section{Results}

The Tables (Tables I and II) demonstrates the mean, standard deviation, and mean differences, as well as the comparison of the variables between pre and posttreatment values of both Orthodontic and Orthognathic groups of patients. The Anthropometric measurements of pre-treatment values when compared to post-treatment values demonstrated significant differences in almost all parameters of the craniofacial region.

The parameters concerning the mouth width, upper and lower lip lengths, upper and lower vermillion heights, mandible ratio, mandible face height ratios showed statistical significance in the orthodontic group $(\mathrm{p} \leq 0.005)$.

The measurements concerning facial height, lower face height, upper and lower lip lengths, lower lip vermillion, mandible to face height ratio showed statistical significance $(p \leq 0.005)$ in Class II skeletal patients (Group II A).

In Class III skeletal patients (Group II B) the parameters concerning facial height, upper facial height, lower lip length, upper and lower lip vermilion, facial index, mandible width- facial height ratio, mandiblelower facial height ratio, and chin-mandible face index showed statistical significance $(\mathrm{p} \leq 0.005)$.

Regarding divine proportions, the parameters that showed statistical significance are alae width-mouth width ratio and upper lip length to lower lip length ratio in Group I.

In the Orthognathic subgroup (Group II A), forehead to eye and eye to menton ratio, upper lip length, and lower lip length ratios showed statistical significance $(p \leq 0.005)$ whereas in the Orthognathic subgroup (Group II B) all the vertical divine proportions showed statistical significance $(p \leq 0.005)$ as shown in Table 2. 


\begin{tabular}{|c|c|c|c|c|c|c|c|c|c|c|c|c|c|c|c|c|c|c|c|c|c|c|c|}
\hline 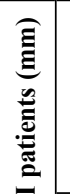 & 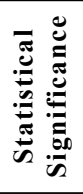 & $\stackrel{m}{n}$ & $\stackrel{\sim}{\sim}$ & ถู & $\begin{array}{l}n \\
\hat{n} \\
\infty\end{array}$ & $\overrightarrow{\widehat{\sigma}}$ & 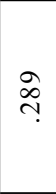 & 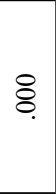 & 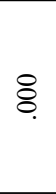 & ڤू & 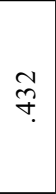 & $\tilde{\delta}$ & ธิ & $\stackrel{t}{\circ}$ & ธิ & ర్t & $\stackrel{n}{n}$ & ¿. & $\overrightarrow{\mathcal{f}}$ & $\stackrel{\infty}{\stackrel{\Gamma}{~}}$ & $\frac{\Im}{0}$ & $\stackrel{t}{\circ}$ & $\stackrel{\circ}{\circ}$ \\
\hline 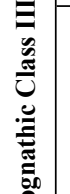 & 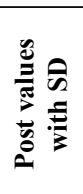 & 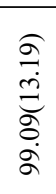 & 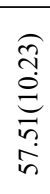 & $\begin{array}{l}\underset{\mathfrak{f}}{\stackrel{N}{n}} \\
\stackrel{n}{n} \\
m \\
m\end{array}$ & 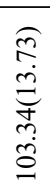 & 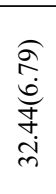 & 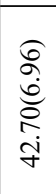 & 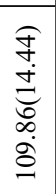 & 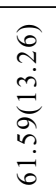 & 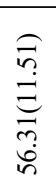 & $\begin{array}{l}\widehat{E} \\
\stackrel{0}{0} \\
\infty \\
\dot{\Xi} \\
\dot{J}\end{array}$ & 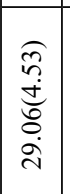 & 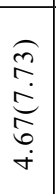 & 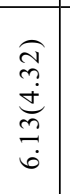 & 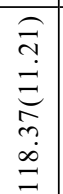 & 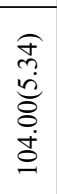 & $\begin{array}{l}\stackrel{\vec{m}}{\stackrel{m}{o}} \\
\stackrel{\infty}{m} \\
\stackrel{m}{r}\end{array}$ & $\begin{array}{l}\hat{\sigma} \\
\infty \\
\stackrel{\sigma}{0} \\
\tilde{m} \\
\dot{\sigma}\end{array}$ & 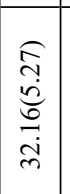 & 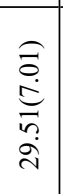 & \begin{tabular}{l}
$\widehat{\partial}$ \\
$\dot{d}$ \\
\multirow{+}{0}{} \\
$\infty$ \\
$\dot{j}$
\end{tabular} & 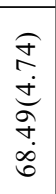 & 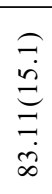 \\
\hline 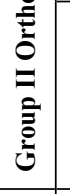 & 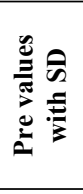 & 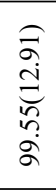 & 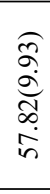 & $\begin{array}{l}\hat{શ} \\
\stackrel{\hat{\sigma}}{0} \\
\infty \\
\dot{m} \\
\dot{m}\end{array}$ & 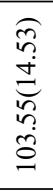 & 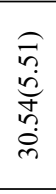 & 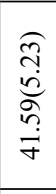 & 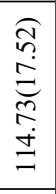 & 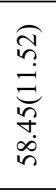 & 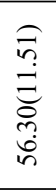 & 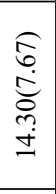 & $\mid \begin{array}{l}\widehat{O} \\
\stackrel{+}{+} \\
\underset{i}{i} \\
i \\
m\end{array}$ & 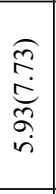 & 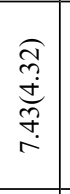 & 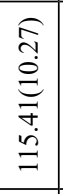 & 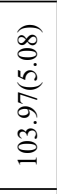 & 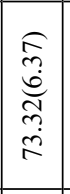 & 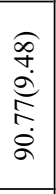 & 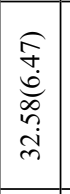 & 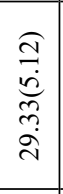 & 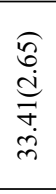 & 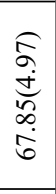 & 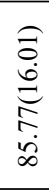 \\
\hline 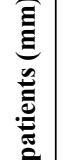 & 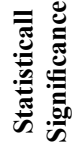 & 今. & $\vec{a}$ & $\stackrel{0}{+}$ & $\stackrel{?}{?}$ & 今े & 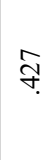 & ț & 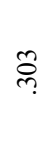 & $\tilde{\delta}$ & $\stackrel{t}{8}$ & 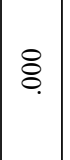 & $\stackrel{8}{:}$ & 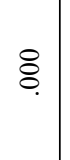 & $\stackrel{\infty}{\text { ஸे }}$ & 宗. & $\stackrel{\nabla}{n}$ & $\begin{array}{l}0 \\
\stackrel{0}{n}\end{array}$ & $\stackrel{\hat{\alpha}}{\infty}$ & 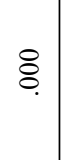 & $\frac{\infty}{a}$ & $\stackrel{\nabla}{\sim}$ & ત્ণે \\
\hline 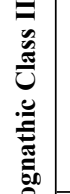 & 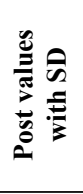 & 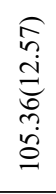 & 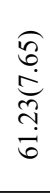 & 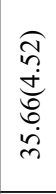 & 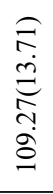 & 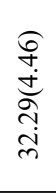 & $\begin{array}{l}\widehat{\sigma} \\
\stackrel{3}{c} \\
\stackrel{0}{0} \\
\dot{+}\end{array}$ & 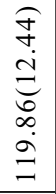 & $\begin{array}{l}\infty \\
\infty \\
\infty \\
\infty \\
\stackrel{0}{0} \\
\stackrel{0}{0}\end{array}$ & 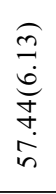 & $\begin{array}{l}\hat{\sigma} \\
0 \\
\dot{\omega} \\
0 \\
\stackrel{n}{2} \\
=\end{array}$ & 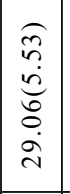 & $\begin{array}{l}\widehat{a} \\
\stackrel{d}{d} \\
\stackrel{b}{r}\end{array}$ & $\begin{array}{l}\underset{J}{d} \\
\stackrel{d}{d} \\
\stackrel{d}{\infty} \\
\infty\end{array}$ & 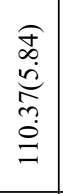 & 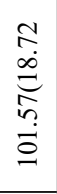 & 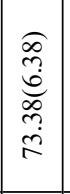 & $\begin{array}{l}\widehat{\sigma} \\
\stackrel{5}{a} \\
\dot{a} \\
a\end{array}$ & 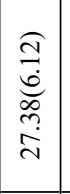 & $\begin{array}{l}\widehat{\jmath} \\
\vec{d} \\
\infty \\
\infty \\
\infty \\
\ddot{d}\end{array}$ & 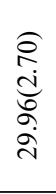 & 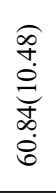 & 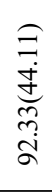 \\
\hline 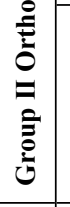 & 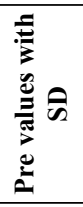 & 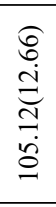 & 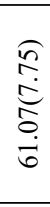 & 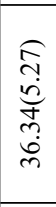 & 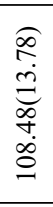 & 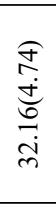 & 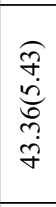 & 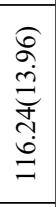 & $\begin{array}{l}\stackrel{f}{m} \\
\stackrel{0}{0} \\
\stackrel{+}{\infty} \\
\stackrel{0}{0}\end{array}$ & 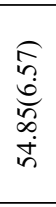 & $\begin{array}{l}\stackrel{0}{0} \\
\vec{c} \\
\stackrel{0}{0} \\
i \\
i\end{array}$ & 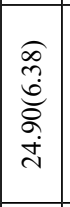 & $\begin{array}{l}\text { 古 } \\
\text { do } \\
\stackrel{0}{\circ} \\
\stackrel{r}{r}\end{array}$ & $\begin{array}{l}\widehat{\infty} \\
\stackrel{n}{d} \\
\stackrel{\infty}{\infty} \\
\stackrel{\infty}{0} \\
\stackrel{0}{0}\end{array}$ & 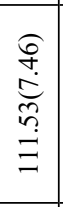 & $\begin{array}{l}\widehat{0} \\
0 \\
0 \\
0 \\
0 \\
0 \\
0 \\
0\end{array}$ & 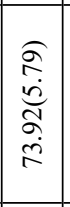 & 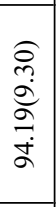 & 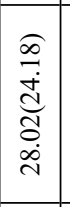 & 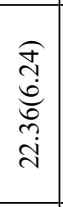 & $\begin{array}{l}\widehat{\sigma} \\
\dot{+} \\
\infty \\
\stackrel{े}{\hat{d}}\end{array}$ & 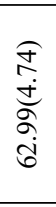 & 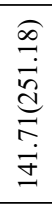 \\
\hline 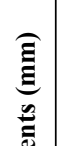 & 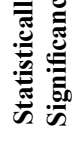 & ț & $\begin{array}{l}\infty \\
\stackrel{0}{0}\end{array}$ & $\cong$ & సે & o̊ & 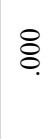 & $\widetilde{f}$ & $\stackrel{t}{i}$ & 斻. & ๕. & 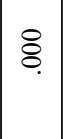 & ธิ & ฮิ & $\vec{\nabla}$ & సे & $\stackrel{0}{\ni}$ & $\stackrel{\infty}{\stackrel{\infty}{m}}$ & 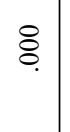 & 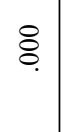 & $\vec{m}$ & $\begin{array}{l}\hat{\infty} \\
\infty \\
\infty\end{array}$ & $\stackrel{\circlearrowright}{\sim}$ \\
\hline 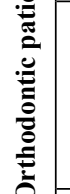 & 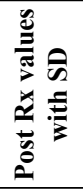 & 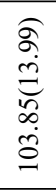 & 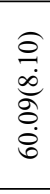 & 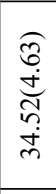 & 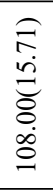 & $\begin{array}{l}\hat{\delta} \\
\dot{0} \\
\dot{0} \\
\infty \\
\dot{0} \\
\dot{m}\end{array}$ & 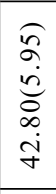 & 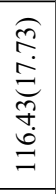 & 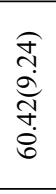 & 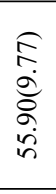 & $\begin{array}{l}\stackrel{o}{\imath} \\
\stackrel{0}{0} \\
\stackrel{0}{0} \\
\stackrel{+}{ \pm}\end{array}$ & 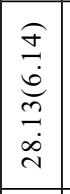 & 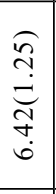 & 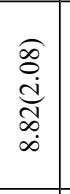 & 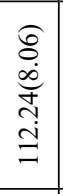 & 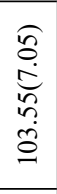 & 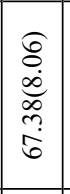 & 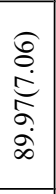 & $\begin{array}{l}\sigma \\
\vec{a} \\
\stackrel{n}{n} \\
m \\
m \\
m\end{array}$ & 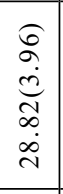 & 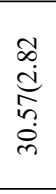 & 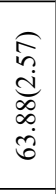 & $\begin{array}{l}\frac{\pi}{0} \\
0 \\
0 \\
0 \\
\dot{0}\end{array}$ \\
\hline 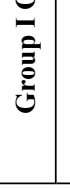 & 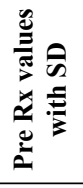 & 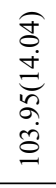 & 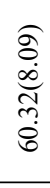 & 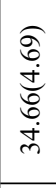 & 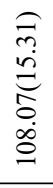 & 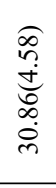 & 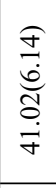 & 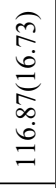 & 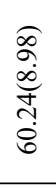 & 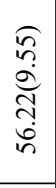 & 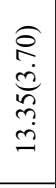 & 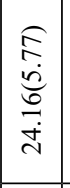 & 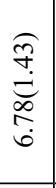 & $\begin{array}{l}\widehat{O} \\
\stackrel{0}{d} \\
\stackrel{d}{n} \\
a\end{array}$ & 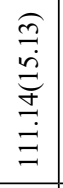 & 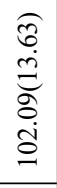 & 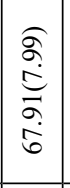 & 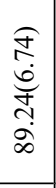 & 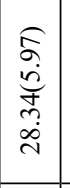 & 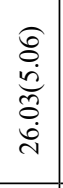 & 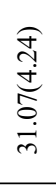 & 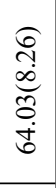 & 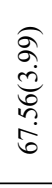 \\
\hline & $\frac{\mathscr{\Xi}}{\pi}$ & $\begin{array}{l}\hat{m} \\
\frac{1}{1} \\
\text { nd }\end{array}$ & $\begin{array}{l}\vec{m} \\
\vec{m}\end{array}$ & ले & $\hat{a}$ & $\begin{array}{l}\vec{m} \\
i \\
i\end{array}$ & $\begin{array}{l}\text { in } \\
1 \\
n \\
n\end{array}$ & 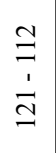 & $\begin{array}{l}\mathbb{1} \\
1 \\
8\end{array}$ & $\begin{array}{l}\text { o } \\
\text { Dे } \\
i n\end{array}$ & $\stackrel{\overbrace{}}{\stackrel{T}{2}}$ & $\begin{array}{l}8 \\
\dot{n} \\
\dot{r}\end{array}$ & $\begin{array}{l}a \\
\infty \\
1 \\
+ \\
\vec{\infty}\end{array}$ & $\begin{array}{l}\stackrel{t}{\circ} \\
\frac{1}{1} \\
\stackrel{a}{a}\end{array}$ & $\begin{array}{l}n \\
\infty \\
\infty \\
1 \\
1 \\
\\
\infty \\
\infty\end{array}$ & $\begin{array}{l}\overrightarrow{0} \\
\stackrel{R}{1} \\
\dot{1} \\
\dot{R}\end{array}$ & $\begin{array}{l}0 \\
\dot{+} \\
\dot{n} \\
\dot{j} \\
i \\
i\end{array}$ & $\begin{array}{l}\text { 官. } \\
\infty \\
1 \\
m \\
\infty \\
\infty\end{array}$ & $\begin{array}{c}\infty \\
\dot{+} \\
1 \\
1 \\
\infty \\
\dot{n}\end{array}$ & $\begin{array}{l}\stackrel{+}{\dot{d}} \\
\stackrel{+}{\sim} \\
\stackrel{\nabla}{\sigma}\end{array}$ & $\begin{array}{l}n \\
8 \\
1 \\
1 \\
\tilde{\delta}\end{array}$ & $\begin{array}{l}\overrightarrow{8} \\
1 \\
1 \\
0 \\
8\end{array}$ & $\begin{array}{l}+ \\
\ddot{j} \\
i \\
\ddot{y} \\
i\end{array}$ \\
\hline & 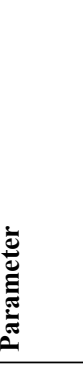 & 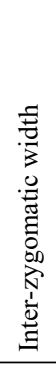 & 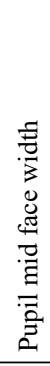 & 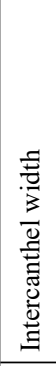 & 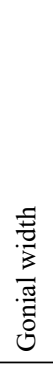 & 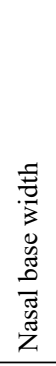 & 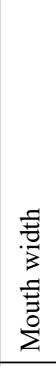 & 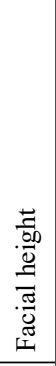 & 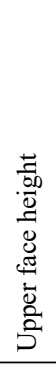 & 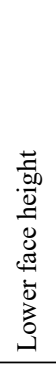 & 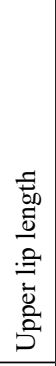 & 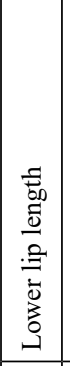 & 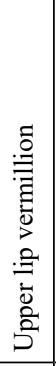 & 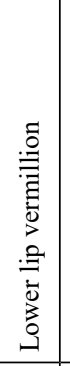 & 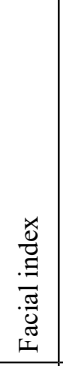 & 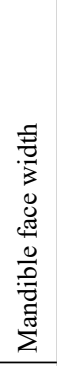 & 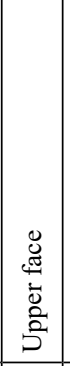 & 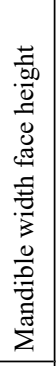 & 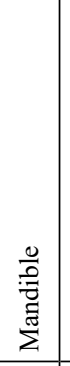 & 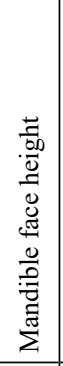 & 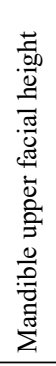 & 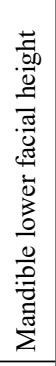 & 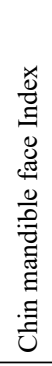 \\
\hline & & - & $N$ & $m$ & $\sigma$ & in & 0 & r & $\infty$ & $a$ & 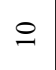 & $=$ & $\simeq$ & 9 & \pm & $\because$ & $\stackrel{0}{0}$ & 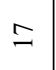 & $\stackrel{2}{ }$ & 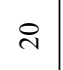 & $\bar{N}$ & $\tilde{ก}$ & $\tilde{\lambda}$ \\
\hline
\end{tabular}




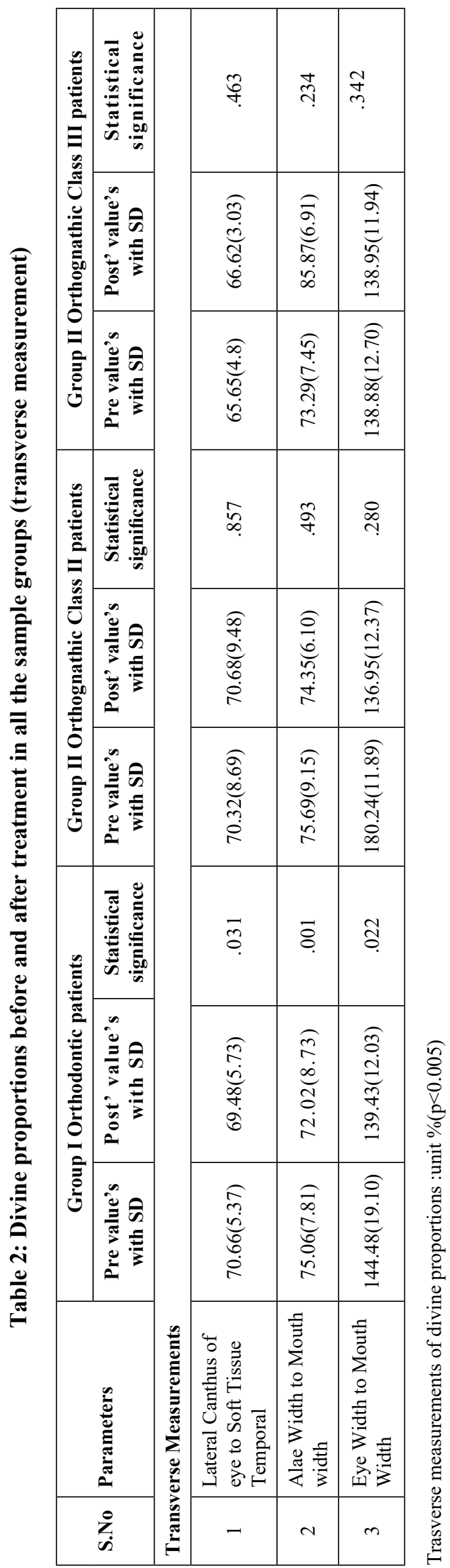

\begin{tabular}{|c|c|c|c|c|c|}
\hline \multirow[t]{3}{*}{$\frac{2}{2}$} & 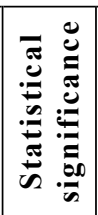 & $\begin{array}{l}\overrightarrow{8} \\
\ddot{8} \\
\ddot{\delta} \\
\varnothing\end{array}$ & $\begin{array}{l}\tilde{\delta} \\
\ddot{\ddot{q}} \\
\stackrel{0}{q}\end{array}$ & $\begin{array}{l}\tilde{\delta} \\
\dot{\theta} \\
\dot{\delta}\end{array}$ & 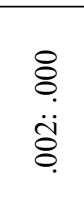 \\
\hline & 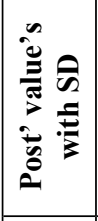 & 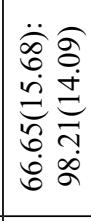 & 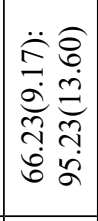 & 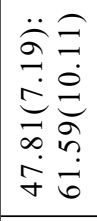 & 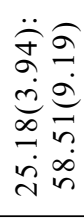 \\
\hline & 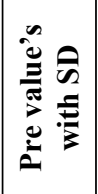 & 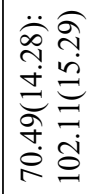 & 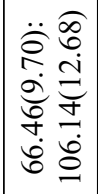 & 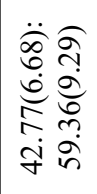 & 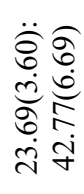 \\
\hline \multirow{3}{*}{ 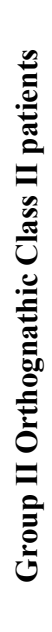 } & 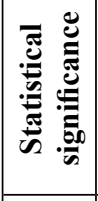 & $\begin{array}{l}\stackrel{\Xi}{8} \\
\ddot{\Xi} \\
\ddot{\Xi}\end{array}$ & $\begin{array}{l}\mathscr{q} \\
\stackrel{+}{0} \\
\ddot{8}\end{array}$ & $\begin{array}{l}\underset{f}{+} \\
\ddot{8} \\
\stackrel{0}{0}\end{array}$ & $\begin{array}{l}\widetilde{8} \\
\ddot{8} \\
\ddot{\delta}\end{array}$ \\
\hline & 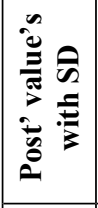 & 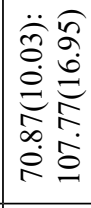 & 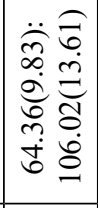 & 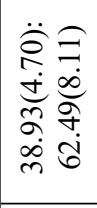 & 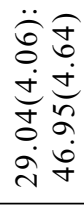 \\
\hline & 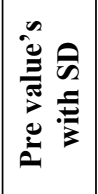 & 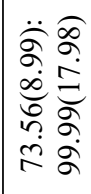 & 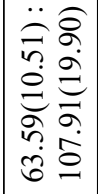 & 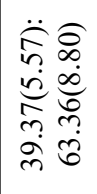 & 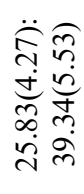 \\
\hline \multirow{3}{*}{ 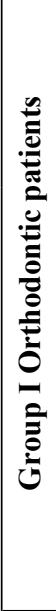 } & 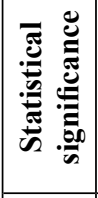 & $\begin{array}{l}\stackrel{0}{0} \\
+ \\
\ddot{q} \\
\dot{q}\end{array}$ & $\begin{array}{l}\stackrel{1}{-} \\
\ddot{0} \\
\grave{\imath}\end{array}$ & $\begin{array}{l}\stackrel{0}{0} \\
\ddot{0} \\
\stackrel{0}{0}\end{array}$ & $\begin{array}{l}\text { oे } \\
\ddot{i} \\
\delta\end{array}$ \\
\hline & 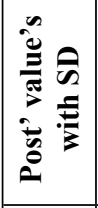 & 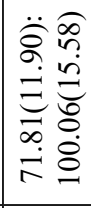 & 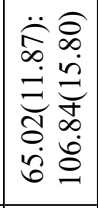 & 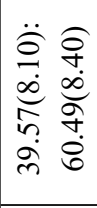 & 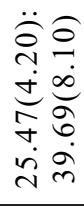 \\
\hline & 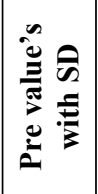 & 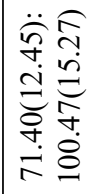 & 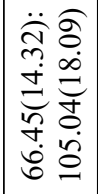 & 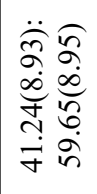 & 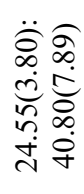 \\
\hline & 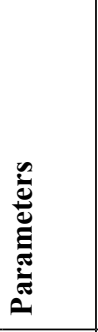 & 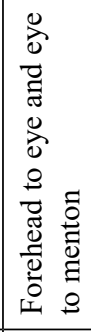 & 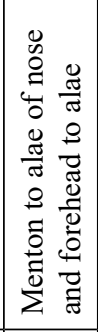 & 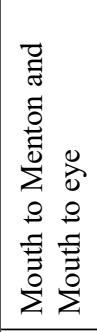 & 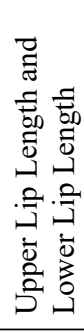 \\
\hline & $\sum_{\dot{n}}^{0}$ & - & $\sim$ & $m$ & $\nabla$ \\
\hline
\end{tabular}




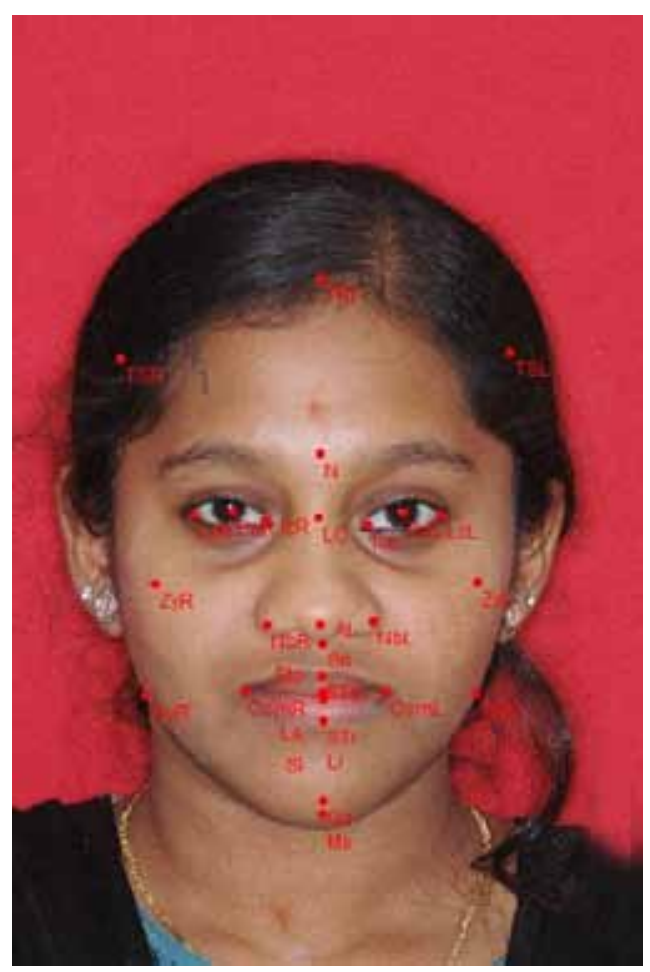

Figure 1: Landmarks (TRI - trichon, TSR - Temporal right, TSL - Temporal left, N- nasion, LC Midpoint of the line drawn from lateral cantheus of the eye, PuR- interpupillary right, PuL - interpupillary left, IcR - inner cantheusof the eye right, IcL-inner cantheus of the left, LcR- lateral cantheus right, LcLlateral cantheus left, ZyR-zygomatic right, ZyL- zygomatic left, Nbr- Nasal base right, Nbl-Nasal base left, AL- alae of the nose, Sn- Subnasale, Sto - stomium, Sts - stomion superior, Sti- stomium inferior, LiLabiale superior, Li- labiale inferior, ComR- Commissure right, ComL- commissure left, SI - sublabial, Gn - gnathion, Me-Menton, GoR- gonial right, GoL- Gonial left.

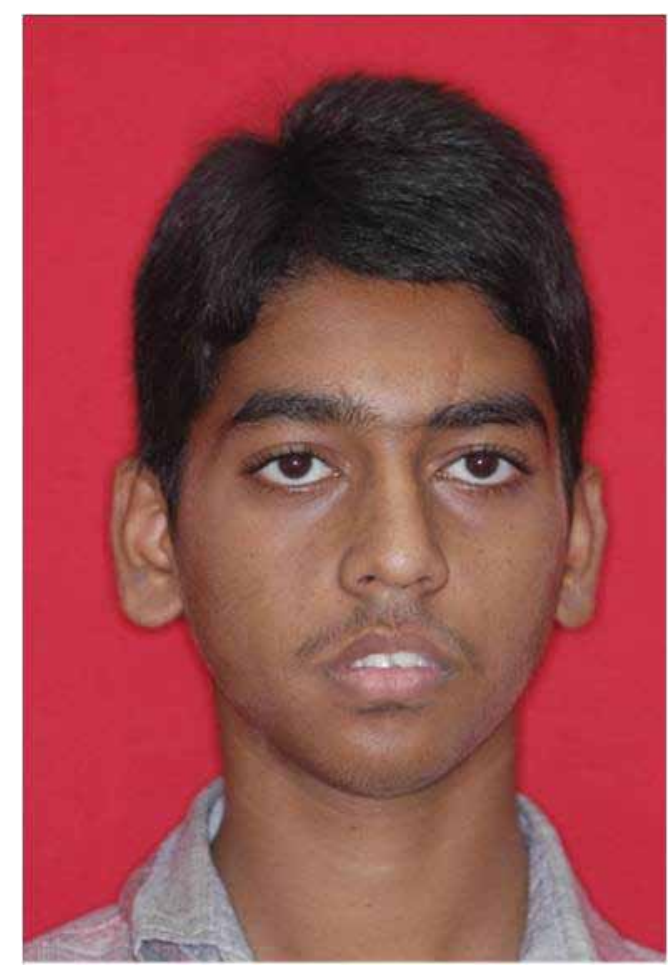

Figure 2a: Sample of Orthodontic group patients(pre) 


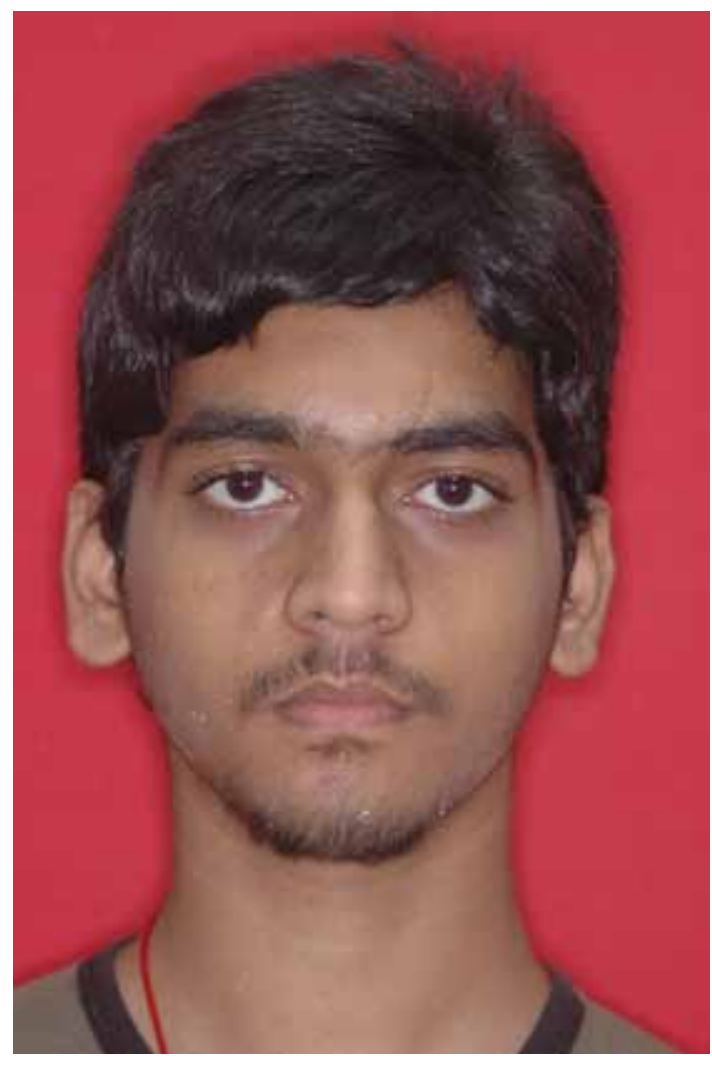

Figure 2b: Sample of Orthodontic group patients(post)

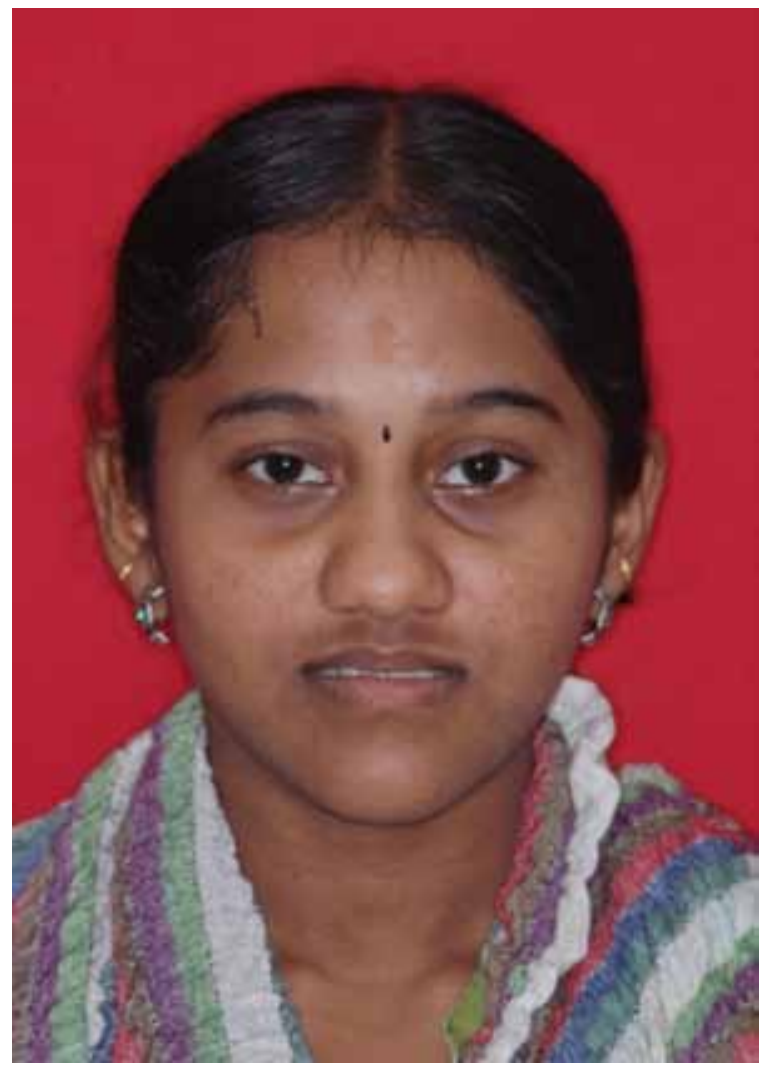

Figure 3a: Sample of Orthognathic (Skeletal Class III) group patients(pre)

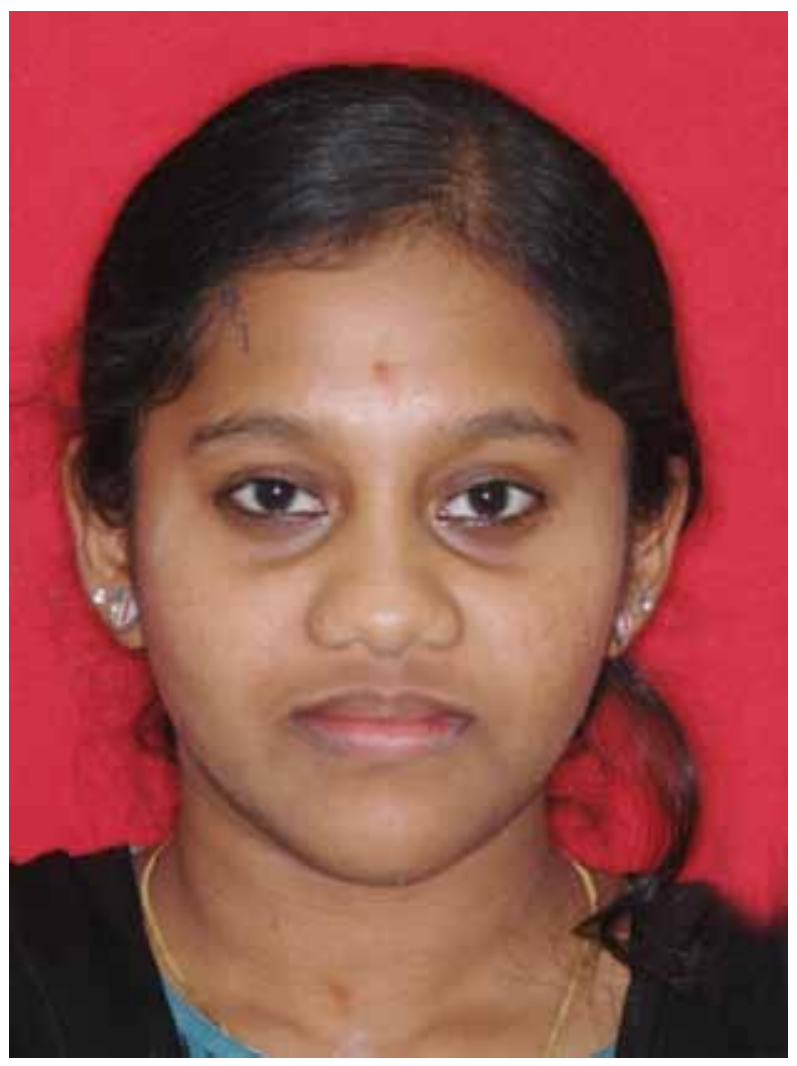

Figure 3b: Sample of Orthognathic (Skeletal Class III) group patients(post)

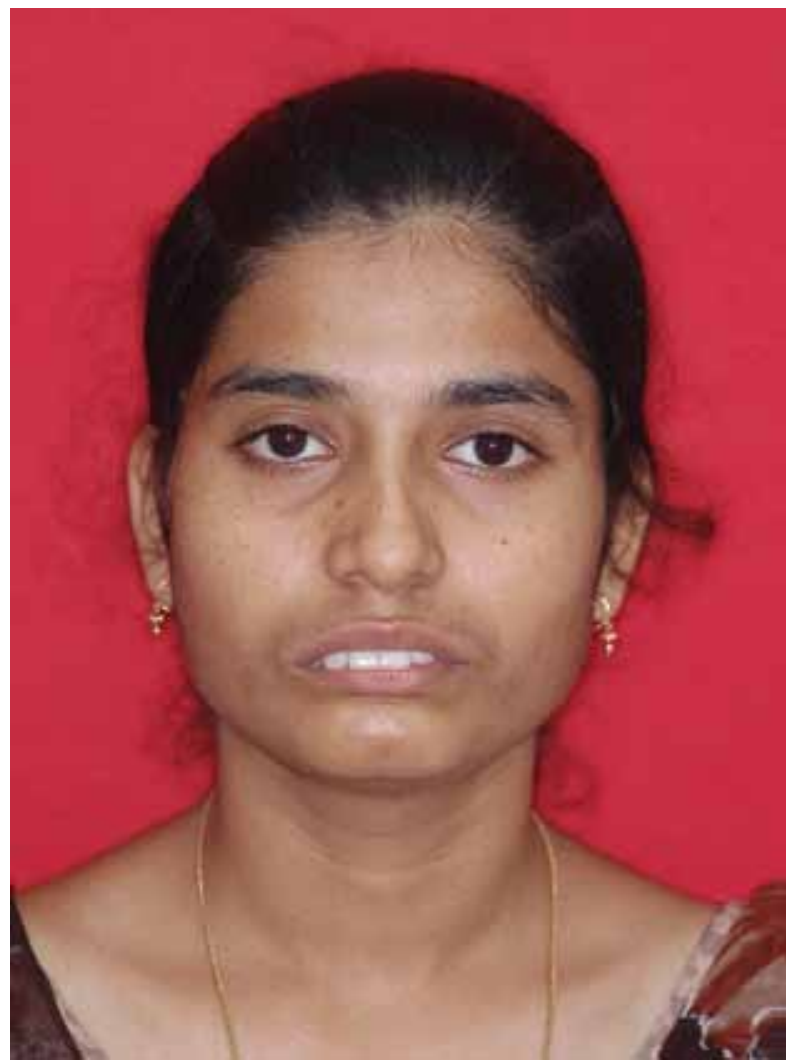

Figure 4a: Sample of Orthognathic (Skeletal Class II) group patients.(pre) 


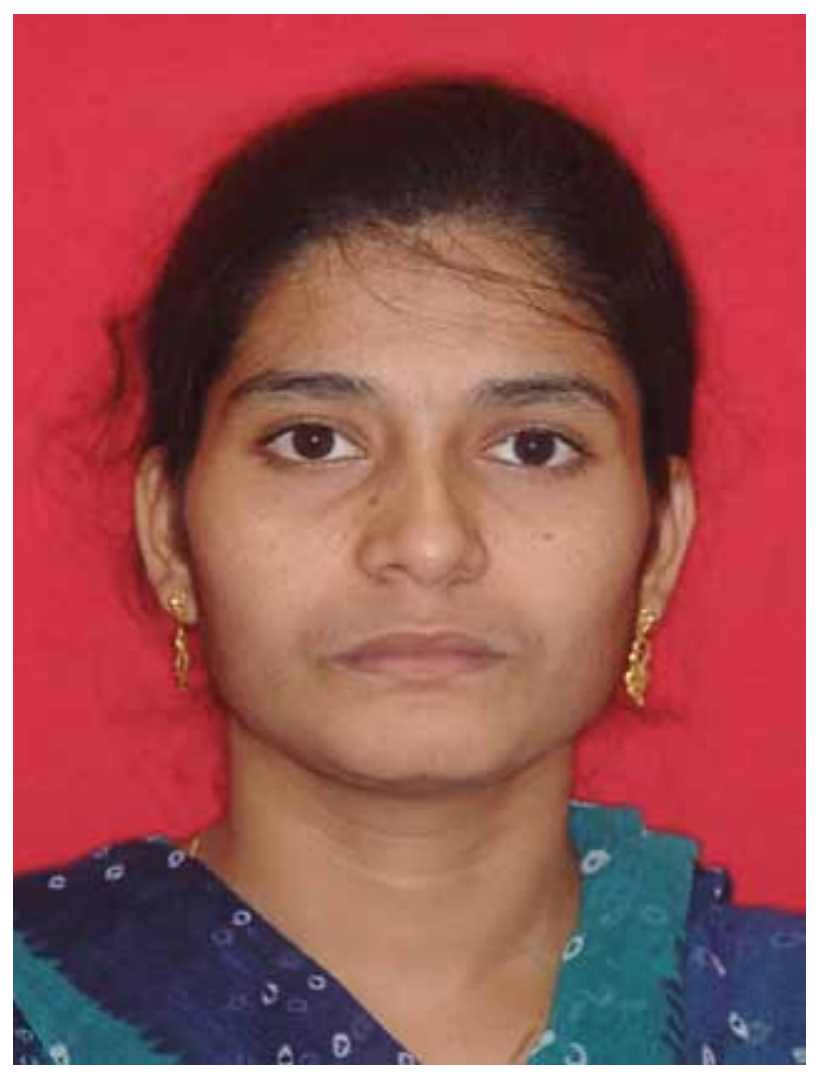

Figure 4b: Sample of Orthognathic (Skeletal Class II) group patients. (post)

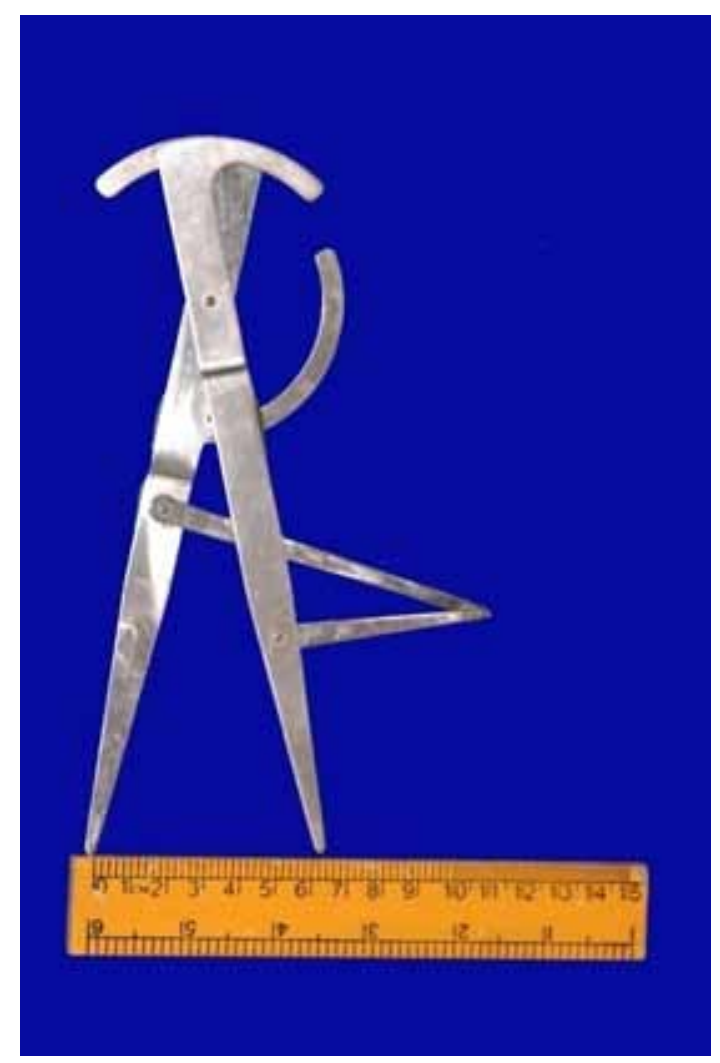

Figure 5a: Measuring the Inter-Pupillary width (instrument)

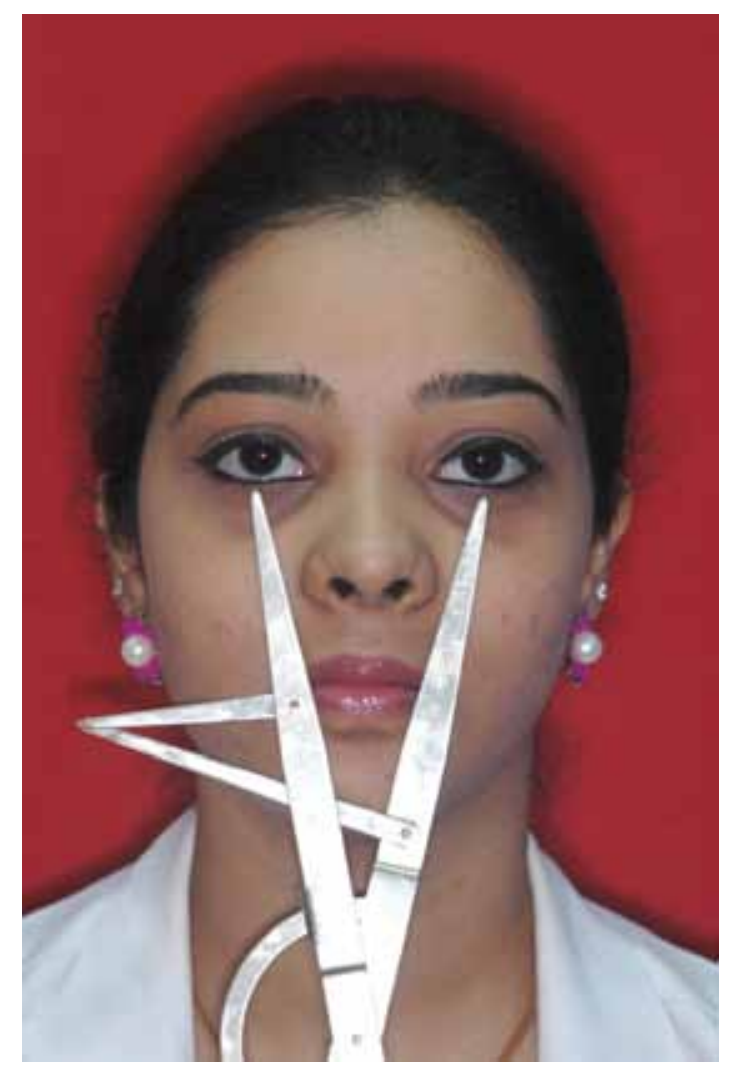

Figure 5b: Measuring the Inter-Pupillary width

\section{Discussion}

The present study aimed to observe the changes in anthropometric measurements post orthodontic and post orthognathic surgery in the Dravidian population. This study also attempted to discuss the proportion achieved after the treatment was nearest to the ratio of indices of golden (divine) proportion $(0.62)^{[2]}$. Considering this, the changes that were observed in the orthodontic, as well as an orthognathic group, resulted in both ratio and percentage format where the value of ratios are represented by a mathematical assumption of $0.6: 1$. According to rickets et al, the ratio of $0.6: 1$ symbolizes the golden proportion which the standard for an aesthetically attractive profile. The maximum changes in the anthropometric parameter were observed in an orthodontic group, statistical significance was upper lip length, lower lip length, upper vermillion show, lower vermillion which was in concordance with a study done by Benedito V. Freitas et al ${ }^{[17]}$.

In the orthodontic group, divine proportion ratio2 changes are evident in alae width and mouth width caused due to the reduction in proclination of the anterior teeth which bring the value closer to the divine proportion ratio $(0.6)$. The other parameter that showed 
significant alteration is the ratio of the upper lip to lower lip length. This change was attributed to the proclination and bite correction which in turn increases the lip length thus bringing it closer to the value of pi or golden proportion. According to Jovana Milutinović, ${ }^{[18]}$ lower facial parameters were approaching the set of divine proportions which is per this study.

In the present study when the Class II orthognathic group was considered, the changes in the chin and convexity were improved due to surgery. The other noticeable changes observed were an increase in total facial height, Lower Face height, upper lip length, lower lip length, and lower vermillion show. These changes were attributed to the advancement of the mandible in a downward and forward direction achieved by Bilateral Sagittal Split Osteotomy (BSSO) which led to a better facial profile and bringing these parameters closer to the ideal value of anthropometric measurements. Whereas, the increase in upper lip length is attributed to a reduction in proclination of the upper anterior teeth. which is in accordance with Sílvia Augusta Braga Reis et al ${ }^{[19]}$ who stated that facial profile convexity and chin prominence are the most important factors which influence facial attractiveness.

In Class II orthognathic patients, the changes in divine proportions were evident in the eye to menton which is a vertical parameter brought about by the BSSO procedure. This is because the mandible is displaced forward owing to the increase in lower face vertical height thus changing the ratio of the eye to menton and forehead to eye achieving a divine proportion. These changes that are observed in the orthognathic group are also in concurrence with the study reported by Siamak Hemmatpour. ${ }^{[21]}$ Also the upper lip length and lower lip length ratios were altered due to an increase in lower face height by mandibular advancement that caused a stretch in the soft tissue drape of the lower face thus establishing golden proportion ratio of $0.6: 1$.

In class III orthognathic patients, the changes in Anthropometric measurements were evident in total facial height, upper face height, lower lip length, upper vermillion show, lower vermillion show. These apparent changes were attributed to the downward and forward movement of the Maxilla through Lefort I osteotomy in combination with an upward and backward displacement of the Mandible due to Mandibular set back (BSSO). The combination or single jaw surgery may vary based on the malocclusion and deformities of the individual's deficiencies where the facial heights are altered. This profile improvement gives the patient a good facial appearance and also brings the linear and angular measurements of the patient's facial landmarks closer to that of Anthropometric measurements. ${ }^{[23]}$

The Indices and ratios in Class III orthognathic patients revealed changes in Facial index, Mandibular width face height, Mandibular lower facial height, Chin Mandible face index. Post-surgical facial height alteration influences all the above-mentioned indices. According to Raymond Edler [26] Orthognathic surgery showed dramatic changes in the soft tissue profile and also anthropometric measurements and indices showed good repeatability in terms of clinical assessment, photography, and digitization to compare these changes that are acquired through orthognathic surgical treatment modalities.

Divine proportions in Class III orthognathic patients revealed evident changes in the parameters such as forehead to eye and eye to menton. These changes are due to the facial height changes caused by the Maxillary and Mandibular surgery (combination or non- combinations) thus achieving a golden proportion in vertical parameters. Secondly, forehead to nose and nose to menton showed changes due to Maxillary advancement (Lefort I osteotomy) which brought about facial changes closer to the golden proportion. The third parameter which was altered was mouth to eye and mouth to menton, owing to the alteration of the facial height. Finally, the ratio of the upper lip to lower lip length was altered due to changes observed in lower face height due to mandibular setback causing an alteration in the soft tissue drape. According to Baker BW et al ${ }^{[27]}$ orthognathic and orthodontic treatment results in an improvement of overall esthetics of patients and also the proportions were equally likely to move away from or toward the divine proportion.

Though significant changes were noticed in both the groups, orthognathic patients revealed higher significance due to the changes in skeletal parameters thereby altering soft tissue drape resulting in values nearer to ideal anthropometric norms.

However, the study would have elicited more accurate results if $3 \mathrm{D}$ software was used. The future scope of the study would be assessing the anthropometric values and divine proportions by involving corrections in patients with craniofacial deformity and asymmetry. 


\section{Conclusion}

1. Both orthodontic and orthognathic patients showed evident facial changes in vertical proportions posttreatment.

2. Orthodontic patients exhibited significant changes concerning mouth width ratios due to the retraction of the anterior teeth.

3. Orthognathic patients unveiled more significant vertical facial changes owing to the alteration of the skeletal bases deriving proportions nigh anthropometric \& facial indices.

Ethical Clearance: Taken from Meenakshi Ammal Dental College

Source of Funding: Meenakshi Academy of Higher Education and Research, Chennai, India

\section{Conflict of Interest: Nil}

\section{References}

1. Baumgarten AG. Aesthetica. Paris: L'herne; 1989.

2. Ricketts RM. The biologic significance of the divine proportion and Fibonacci series. American journal of orthodontics. 1982 May 1;81(5):351-70.

3. Farkas LG, Bryson W, Klotz J. Is photogrammetry of the face reliable?. Plastic and Reconstructive surgery. 1980 Sep;66(3):346-55.

4. Van der Geld P, Oosterveld P, Van Heck G, Kuijpers-Jagtman AM. Smile attractiveness: selfperception and influence on personality. The Angle Orthodontist. 2007 Sep;77(5):759-65.

5. Farkas LG, Munro IR, editors. Anthropometric facial proportions in medicine. Charles $\mathrm{C}$ Thomas Pub Limited; 1987.

6. Lucas WP, Pryor HB. Range and standard deviations of certain physical measurements in healthy children. The Journal of Pediatrics. 1935 Apr 1;6(4):533-45.

7. Milutinovic J, Zelic K, Nedeljkovic N. Evaluation of facial beauty using anthropometric proportions. The Scientific World Journal. 2014 Jan 1;2014.

8. Bengel W. Standardization in dental photography. International Dental Journal. 1985 Sep 1;35(3):2107.

9. Hussain MW, Qamar K, Naeem S. THE ROLE OF INTERPUPILLARY DISTANCE IN THE SELECTION OF ANTERIOR TEETH. Pakistan
Oral \& Dental Journal. 2012 Jun 1;32(1).

10. Bindra B, Basker RM, Besford JN. A study of the use of photographs for denture tooth selection. International Journal of Prosthodontics. 2001 Mar $1 ; 14(2)$.

11. Langlois $\mathrm{JH}$, Roggman LA. Attractive faces are only average. Psychological science. 1990 Mar;1(2):115-21.

12. Perrett DI, May KA, Yoshikawa S. Facial shape and judgements of female attractiveness. Nature. 1994 Mar;368(6468):239-42.

13. Varela M, Garcia-Camba JE. Impact of orthodontics on the psychologic profile of adult patients: a prospective study. American Journal of Orthodontics and Dentofacial Orthopedics. 1995 Aug 1;108(2):142-8.

14. Ras F, Habets LL, Van Ginkel FC, PrahlAndersen B. Quantification of facial morphology using stereophotogrammetry-demonstration of a new concept. Journal of Dentistry. 1996 Sep 1;24(5):369-74.

15. Ferring V, Pancherz H. Divine proportions in the growing face. American Journal of Orthodontics and Dentofacial Orthopedics. 2008 Oct 1;134(4):472-9.

16. Pancherz H, Knapp V, Erbe C, Heiss AM. Divine proportions in attractive and nonattractive faces. World journal of orthodontics. 2010 Mar 1;11(1).

17. Freitas BV, Rodrigues VP, Rodrigues MF, de Melo HV, dos Santos PC. Soft tissue facial profile changes after orthodontic treatment with or without tooth extractions in Class I malocclusion patients: A comparative study. Journal of oral biology and craniofacial research. 2019 Apr 1;9(2):172-6.

18. Milutinović J, Nedeljković N. En-face parameters change after orthodontic treatment of Class II malocclusion. Stomatološki glasnik Srbije. 2016;63(4):167-75.

19. Reis SA, Abrão J, Claro CA, Capelozza Filho L. Evaluation of the determinants of facial profile aesthetics. Dental Press Journal of Orthodontics. 2011 Feb;16(1):57-67.

20. Mesaros A, Cornea D, Cioara L, Dudea D, Mesaros M, Badea M. Facial attractiveness assessment using illustrated questionnairers. Clujul Medical. 2015;88(1):73.

21. Hemmatpour S, Oliadarani FK, Hasani A, Rakhshan V. Frontal-view nasolabial soft tissue 
alterations after bimaxillary orthognathic surgery in class III patients. Journal of Orofacial Orthopedics/ Fortschritte der Kieferorthopädie. 2016 Nov 1;77(6):400-8.

22. August 2016

23. Akan S, Torgut AG, Oktay H. Effects of malocclusions on facial attractiveness and their correlations with the divine proportion. Journal of Orofacial Orthopedics/Fortschritte der Kieferorthopädie. 2017 Sep 1;78(5):427-36.

24. Pettijohn TF, Jungeberg BJ. Playboy playmate curves: Changes in facial and body feature preferences across social and economic conditions. Personality and Social Psychology Bulletin. 2004 Sep;30(9):1186-97.

25. Naini FB, Moss JP, Gill DS. The enigma of facial beauty: esthetics, proportions, deformity, and controversy. American Journal of Orthodontics and Dentofacial Orthopedics. 2006 Sep 1;130(3):27782.

26. Batwa $\mathrm{W}$. The influence of the smile on the perceived facial type esthetics. BioMed research international. 2018 Jan 1;2018.

27. Edler R, Agarwal P, Wertheim D, Greenhill D. The use of anthropometric proportion indices in the measurement of facial attractiveness. The European Journal of Orthodontics. 2006 Jun 1;28(3):274-81.

28. Baker BW, Woods MG. The role of the divine proportion in the esthetic improvement of patients undergoing combined orthodontic/orthognathic surgical treatment. The International journal of adult orthodontics and orthognathic surgery. 2001; 16(2):108-20. 